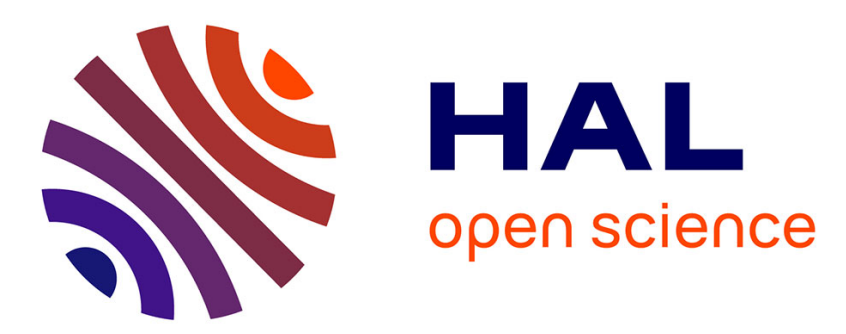

\title{
Thermal response of hyperelastic materials under cyclic loading conditions: specificities and consequences for fatigue
}

\author{
Xavier Balandraud, Jean-Benoit Le Cam
}

\section{- To cite this version:}

Xavier Balandraud, Jean-Benoit Le Cam. Thermal response of hyperelastic materials under cyclic loading conditions: specificities and consequences for fatigue. European Conference on Constitutive Models for Rubber VIII, Jun 2013, San Sebastian, Spain. hal-01136530

\author{
HAL Id: hal-01136530 \\ https://hal.science/hal-01136530
}

Submitted on 18 May 2020

HAL is a multi-disciplinary open access archive for the deposit and dissemination of scientific research documents, whether they are published or not. The documents may come from teaching and research institutions in France or abroad, or from public or private research centers.
L'archive ouverte pluridisciplinaire HAL, est destinée au dépôt et à la diffusion de documents scientifiques de niveau recherche, publiés ou non, émanant des établissements d'enseignement et de recherche français ou étrangers, des laboratoires publics ou privés. 


\title{
Thermal response of hyperelastic materials under cyclic loading conditions: Specificities and consequences for fatigue
}

\author{
X. Balandraud \\ Clermont Université, Institut Français de Mécanique Avancée, Institut Pascal, Clermont-Ferrand, France \\ CNRS, UMR 6602, Institut Pascal, Aubière, France
}

J.-B. Le Cam

Université de Rennes, LARMAUR ERL CNRS 6274, Campus de Beaulieu, Rennes, France

\begin{abstract}
The present paper deals with the specificities of the thermal response of rubber-like materials under mechanical cyclic loading at constant ambient temperature. For this purpose, the framework of thermo-hyper-elasticity is used to predict the heat sources produced or absorbed by the material during stretching due to the entropic coupling. The heat diffusion equation is then used to deduce the temperature variations under non-adiabatic conditions. The influence of several parameters is studied: signal shape, minimum and maximum stretch levels and multiaxiality of the mechanical state. Results show that, in the steady-state regime, the mean value between the maximum and minimum temperature variations over a mechanical cycle is different from zero. This is due to the fact that the heat source depends on both the stretch rate and the stretch level. This result leads to an important consequence for fatigue. Indeed, the stabilized mean value between the maximum and minimum temperature variations during fatigue tests does not reflect only fatigue damage, since the entropic coupling leads also to a value different from zero. This is a major difference with materials exhibiting only an isentropic coupling, for instance metallic materials.
\end{abstract}

\section{INTRODUCTION}

Elastomers are polymeric materials that can undergo large deformations without breaking owing to the ability of their constituent polymeric chains to rotate about the chain bonds. They are used in many application fields as mechanical engineering, automotive engineering and aerospace engineering due to their high elasticity, high damping and high elongation at failure. However, many phenomena involved in the deformation process of such materials are still not really understood. One can cite non-exhaustively phenomena such as: stress softening referred to as the Mullins effect (Mullins 1948; Marckmann et al. 2002; Diani et al. 2009) in the literature, the Payne (or Fletcher-Gent) effect (Fletcher \& Gent 1953; Payne 1962; Barick \& Tripathy 2010; Barmouz et al. 2011), the cavitation (Stringfellow \& Abeyaratne 1989; Le Cam \& Toussaint 2008; Le Cam \& Tousaint 2009) and the stress-induced crystallization (Toki et al. 2000, Toki et al. 2002; Trabelsi et al. 2002; Trabelsi et al. 2003; Huneau 2011). Classically, rubber-like elasticity is described as an entropic effect, which leads to a heat production (absorption) during loading (unloading). The first experimental observations of this effect in the literature were those obtained by Gough (1805) and Joule (1857), which dealt with the increase in temperature of rubber during stretching. In spite of these first results, no further experimental study was fully dedicated to the thermomechanical characterization of the elastomers, although this is a key-point to study and to predict phenomena such as the fatigue damage (Mars \& Fatemi 2002a; Mars 2002b; Le Cam 2005; Saintier et al. 2006), and to establish material constitutive equations. In a theoretical point of view, the work by Chadwich and Creasy (1984) is one of the very few studies that echoes the experimental study of Joule. Up to day, no study allows us to link this theoretical modeling with experimental results, even for homogeneous thermomechanical fields.

The present study deals with the predicted temperature evolution of rubber under cyclic loading at constant ambient temperature. The heat sources produced or absorbed by the stretched material are obtained from the loading conditions by using the framework of the continuum thermodynamics (Lemaitre \& Chaboche 1990; Maugin 1999) with an isotropic thermo-hyper-elastic behaviour. The heat sources considered here are only due to the entropic coupling. The temperature variations are then 
deduced from the heat sources by solving the heat diffusion equation. For the sake of simplicity of processing (in order to avoid full 3D calculations), applications are performed here with homogeneous deformation fields. This hypothesis does not alter the conclusions of the study and enables us to focus on the specificities of the thermal response of rubber.

The paper is composed of two sections. The modeling and the numerical simulation set-up are presented in Section 2. In particular, the hypotheses to predict the temperature evolution are given, as well as the loading conditions of the cyclic tests to be simulated. Section 3 is devoted to the analysis of the results. In particular, we focus on the steadystate temperature evolution.

\section{MODELLING AND NUMERICAL SIMULATION SET-UP}

This section presents the expression of the heat diffusion equation that is used, the expression considered for the heat source, and the different tests to be simulated.

\subsection{Heat diffusion equation}

Some assumptions are used to simplify the heat diffusion equation in case of homogeneous deformation fields. These assumptions aims at simplifying the calculations and do not alter the conclusions of the study.

Let us consider a mechanical loading such that the deformation field is homogeneous in the specimen at any time. Due to the very small thermal diffusivity of elastomers (order of magnitude of $1 \mathrm{E}-7 \mathrm{~m}^{2} / \mathrm{s}$ ), the temperature field is almost homogeneous in the specimen at any time. Let us denote by $\theta$ the temperature variation with respect to the temperature in the reference state, taken in the undeformed state. The heat exchanges at the specimen boundary are linearized assuming that the temperature variations $\theta$ are small. After some calculations not detailed here, the heat diffusion equation can be rewritten as follows (Chrysochoos et al. 1996; Balandraud at al. 1999; Boulanger et al. 2004; Berthel et al. 2008):

$$
\rho C\left(\frac{d \theta}{d t}+\frac{\theta}{\tau}\right)=s_{h}
$$

where $\tau$ is a time constant characterizing the heat exchanges between the specimen and its surroundings. This formulation can be referred to as the " $0 \mathrm{D}$ " formulation. For similar simplifications of the heat diffusion equation, the reader can also refer to Refs. (Chrysochoos \& Louche 2000; Chrysochoos \& Louche 2001; Chrysochoos et al. 2009; Balandraud et al. 2001; Maquin \& Pierron 2009; Dumoulin et al.
2010), which are devoted to wires or thin plates, leading respectively to $1 \mathrm{D}$ or $2 \mathrm{D}$ formulations.

The value of $\tau$ characterizes the level of nonadiabaticity of the mechanical test. If $\tau=0$, the test is isothermal (the heat produced or absorbed by the material due to stretch is instantaneously exchanged with the outside). A perfectly adiabiatic evolution corresponds to $\tau=\infty$. More generally, if $\tau$ is much larger than the test duration, the evolution can be considered as adiabatic over this duration. It is worth noting that, experimentally, $\tau$ depends on the specimen material (through its thermal diffusivity), the environment (ambient air and material used for the grips of the testing machine) and the specimen geometry (through the surface-areato-volume ratio). Let us also note that the change in the specimen shape (due to large deformations) modifies the surface-area-to-volume ratio. In the present numerical study, $\tau$ is considered as a constant. Taking into account its variations does not alter the conclusions of the study.

\subsection{Heat sources due to entropic coupling}

In the present study, the thermodynamical evolution is mechanically purely reversible. In other words, no mechanical dissipation (mechanical dissipation) is produced by the material: $D_{\text {int }}=0$. Moreover, only the effects of the entropic coupling are considered in the heat source expression. Such material behavior is observed for unfilled rubbers, for instance in silicone rubbers that exhibit a perfect non-linear and reversible behavior with no Mullins effect nor hysteresis loop, meaning that loading and unloading curves are superimposed (Meunier et al. 2008).

The prediction of the heat sources produced during the deformation process requires the choice of a free energy density $\Psi$. Here, the material is assumed to behave as a hyperelastic material that is mechanically incompressible. As high strain levels are not investigated here, the Neo-Hookean model is chosen (Treloar 1943). The free energy function is then given by the following strain energy density:

$\rho \Psi(\mathbf{F}, T)=\mathrm{W}(\mathbf{F}, T)=\frac{1}{2} N k T\left(I_{I}-3\right)$

where $N$ is the number of network chains per unit volume, $k$ the Boltzmann's constant, $T$ the temperature in Kelvin and $I_{1}$ the first invariant of the left Cauchy-Green deformation tensor $\mathbf{F} \mathbf{F}^{\mathrm{T}}$. If the material is mechanically incompressible, the deformation gradient tensor for a biaxial loading is given by:

$\mathbf{F}=\lambda \mathrm{e}_{1} \otimes \mathrm{e}_{1}+\lambda^{B} \mathrm{e}_{2} \otimes \mathrm{e}_{2}+\lambda^{-(B+1)} \mathrm{e}_{3} \otimes \mathrm{e}_{3}$

where

- $\mathrm{e}_{1}, \mathrm{e}_{2}$ and $\mathrm{e}_{3}$ are three orthonormal vectors of the 3D Euclidian space, 
- $\lambda$ is the stretch ratio in the $\mathrm{e}_{1}$ direction, defined as the current length to the initial length ratio in the $\mathrm{e}_{1}$ direction,

- the operator $\otimes$ between two vectors is such that $[\mathrm{a} \otimes \mathrm{b}]_{i j}=a_{i} b_{j}$.

$B$ is the coefficient of biaxiality. It is equal to -0.5 , 0 and 1 for Uniaxial Tension (UT), Pure Shear (PS) and Equibiaxial Tension (EQT), respectively.

The strain energy density writes:

$$
W(\lambda, T)=\frac{1}{2} N k T\left[\lambda^{2}+\lambda^{2 B}+\lambda^{-2(B+1)}-3\right]
$$

The heat source is then given by:

$$
s_{h}=T \frac{\partial^{2} W}{\partial T \partial \lambda} \times \frac{d \lambda}{d t}
$$

Using this expression, the heat diffusion equation writes:

$$
\begin{aligned}
\rho C & \left(\frac{d \theta}{d t}+\frac{\theta}{\tau}\right) \\
& =N k T\left[\lambda+B \lambda^{2 B-1}-(B+1) \lambda^{-2 B-3}\right] \frac{d \lambda}{d t}
\end{aligned}
$$

Eq. 6 is used below to predict the thermal response of rubber for different loading conditions.

Remark 1: the heat source due to the entropic coupling depends on the stretch rate $d \lambda / d t$, but also on the stretch ratio through the term $\lambda+B \lambda^{2 B-1}-(B+1) \lambda^{-2 B-3}$. This is a major difference with materials exhibiting only an isentropictype thermoelastic coupling (metallic materials in particular). In this latter case, the heat source depends only on the strain rate. This property has inspired the so-called Thermoelastic Stress Analysis (TSA) technique, which enables one to measure the stress distribution in metallic structural components from full temperature field measurements.

Remark 2: the temperature variation induces also a variation of the heat source through the term $N k T$. However, this effect is negligible because $T$ is expressed in Kelvin. For instance, a temperature variation of 1 degree leads to a source variation of $100 \times 1 / 273=0.37 \%$. This negligible phenomenon is not specific to materials exhibiting an entropic coupling. It exists also for metallic materials for instance. For the present study, $T$ is kept constant and equal to the ambient temperature $293 \mathrm{~K}$.

\subsection{Simulations}

This section describes the different simulation tests and provides some information about the numerical resolution of the heat diffusion equation.

Material parameters: the material is assumed to behave as a neo-hookean material. In this case, the only material parameter is the number of the chain segments $N$ per unit volume. In the simulations below, the quantity $N k T$ is kept constant (see Remark 2 above). It is taken equal to $2 \mathrm{MPa}$.

Level of non-adiabaticity of the thermodynamical evolution: the time constant $\tau$ is taken equal to $60 \mathrm{~s}$. This is the order of magnitude of $\tau$ measured for a $1 \times 5 \times 20 \mathrm{~mm}^{3}$ natural rubber specimen (experiments not reported here).

Loading conditions: cyclic tests are considered here. Several parameters are studied: shape of the signal applied, strain ratios and multiaxiality of the mechanical state. Two types of signal are considered: triangular and sinusoidal. The former case enables us to exclude the influence of the stretch rate variation from the analysis $(d \lambda / d t$ is constant during the loading stage and the unloading stage). The latter case is classically used for experimental cyclic tests. Table 1 gives the different tests to be carried out. The first two columns give the minimum and maximum stretch ratios applied, $\lambda_{\min }$ and $\lambda_{\max }$ respectively. The strain ratio $R_{\varepsilon}$ is defined as the ratio between the minimum strain and the maximum strain. $f$ is the frequency of the mechanical cyclic loading.

- Tests \#1 to \#3 enable us to study the effect of the loading parameters in case of UT.

- Tests \#4 and \#5 enable us to compare with EQT and PS.

The test duration is chosen equal to $300 \mathrm{~s}$ for all tests, which corresponds to $5 \tau$. This enables us to observe both the transient and stabilized regimes.

Data to be extracted from the temperature evolutions: one of the objectives is to study the stabilized thermal response, which is obtained after a certain duration (the stabilized evolution is reached after a duration which can be approximated to about $3 \tau$ ). Under non-adiabatic conditions, the thermal response evolves from one cycle to another before stabilizing. In order to follow the global thermal response, we define by $\hat{\theta}$ the mean value between the maximum and minimum temperature variations over a mechanical cycle by:

$\hat{\theta}=\frac{\theta_{\min }+\theta_{\max }}{2}$

where $\theta_{\min }$ and $\theta_{\max }$ are the minimum and maximum temperature variations over a mechanical cyclic.

Numerical resolution: the resolution of Eq. 6 is based on an implicit Euler scheme. A particular attention is paid on the time discretization. Accurate results require a very refined temporal 
Table 1. Mechanical tests.

\begin{tabular}{lllllll}
\hline Test number & $\lambda_{\min }-\lambda_{\max }$ & $R_{\varepsilon}$ & $f(\mathrm{~Hz})$ & $\tau(\mathrm{s})$ & Signal shape & Loading shape \\
\hline$\# 1$ & $1-3$ & 0 & 0.5 & 60 & Triangular & UT \\
$\# 2$ & $1-3$ & 0 & 0.5 & 60 & Sinusoidal & UT \\
$\# 3$ & $2-4$ & $1 / 2$ & 0.5 & 60 & Sinusoidal & UT \\
$\# 4$ & $1-3$ & 0 & 0.5 & 60 & Sinusoidal & EQT \\
$\# 5$ & $1-3$ & 0 & 0.5 & 60 & Sinusoidal & PS \\
\hline
\end{tabular}

discretization. The number of time steps per mechanical cycle is imposed. After some preliminary tests, this number is fixed to $1 \mathrm{E} 4$. Such a refined temporal discretization is particularly required for the triangular loading, which exhibits a non-continuous evolution of the heat source, induced by the non-continuous evolution of $d \lambda / d t$.

Remark about the heat sources: the heat source $s_{h}$ is expressed in $\mathrm{W} / \mathrm{m}^{3}$. However, it can be useful to divide this quantity by $\rho C$ :

$s=\frac{s_{h}}{\rho C}$

In the following of the paper, the term "heat source" is still used for the quantity $s$. This latter is expressed in ${ }^{\circ} \mathrm{C} / \mathrm{s}$. Using this unit enables us to directly read the temperature rate in case of an adiabatic evolution. Obviously, in case of non-adiabatic conditions, the temperature rate is different from the heat source $s$, due to the heat exchanges with the outside. The heat diffusion equation becomes:

$\frac{d \theta}{d t}+\frac{\theta}{\tau}=s$

Note finally that the term "heat" must be distinguished to the term "heat source". The heat is the temporal integration of the heat sources. It is expressed in $\mathrm{J} / \mathrm{m}^{3}$ (in ${ }^{\circ} \mathrm{C}$ when divided by $\rho C$ ).

\section{RESULTS AND ANALYSIS}

\subsection{Study of the UT tests}

Figure 1 presents the evolution of the temperature variation for a triangular signal under nonadiabatic condition (test \#1). Two main results can be drawn from this figure:

First, the quantity $\hat{\theta}$, which corresponds to the mean value between the maximum and minimum variations over a mechanical cycle, decreases over the test duration. This can be simply explained as follows. During the first loading stage, the temperature increases because the heat source is positive. However, a certain quantity of heat is given to the outside because the material is warmer than the outside (this heat corresponds to the temporal integration of $\theta / \tau)$. So, the temperature at the end of the first loading stage is lower than the value that would be obtained for adiabatic test conditions. During unloading, the heat source is negative, leading to a temperature decrease. This phenomenon is amplified by the fact that the material continues to give heat to the outside as long as it is warmer than the outside (as long as $\theta / \tau$ is positive). As a consequence, the temperature variation at the end of the first mechanical cycle is lower than zero. This phenomenon goes on over the cycles.

Second, it can be observed that $\hat{\theta}$ reaches a stabilized value which is different from zero: see Figure 1-b which shows the last two stabilized cycles (at the end of test \#1). Here, $\hat{\theta}$ is equal $0.4043^{\circ} \mathrm{C}$. This effect is due to the particular evolution of the heat source in time (see Remark 1 in Section 2.2). This is a significant result, typical of rubber-like materials. It is worth noting that this phenomenon is not due to the mechanical dissipation ( $D_{\text {int }}$ equal to zero in the simulations). Note also that this phenomenon is observed while the temporal integration of the heat source is null over each cycle.

This result has important consequences for fatigue prediction. Indeed, the stabilized mean value between the maximum and minimum temperature variations during experimental fatigue tests does not reflect only fatigue damage, since the entropic coupling leads also to a value that is not equal to zero.

Remark: unlike $\hat{\theta}$, the mean temperature variation over one cycle (defined as the temporal integration of $\theta$ over one cycle) is equal to zero in the steadystate regime. Indeed, the temporal integration of the heat source $s$ over one mechanical cycle is equal to zero; in the steady-state regime, the temporal integration of $d \theta / d t$ is also equal to zero. As $\theta=\tau \times(s-d \theta / d t)$ according to Eq. 9, the mean temperature variation over one cycle is equal to zero in the steady-state regime.

Test \#2 corresponds to the same parameters as for the previous test, but with a sinusoidal signal. 


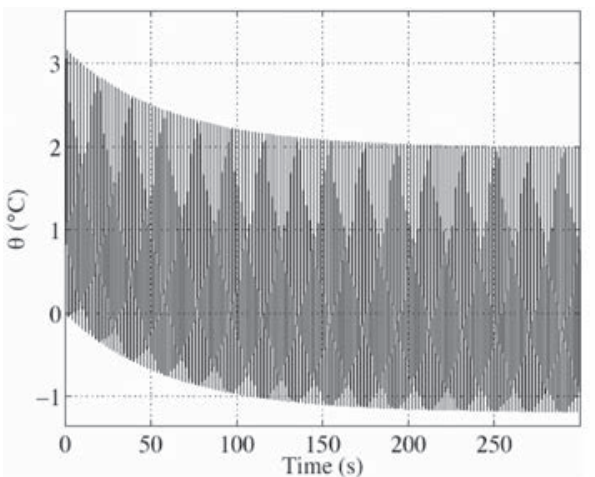

(a)
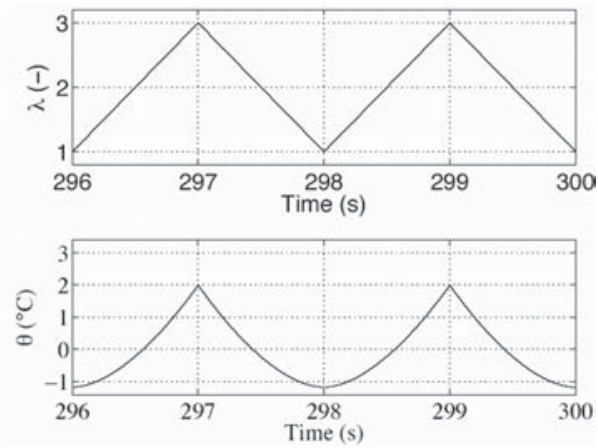

(b)

Figure 1. Test \#1. (a) Temperature variation vs. time. (b) Last two cycles.

Figure 2 gives the evolution of the temperature variation $\theta$ for two stabilized cycles. As observed previously for the triangular signal shape, the value of $\hat{\theta}$ is different from zero in the steadystate regime. Moreover, the stabilized value of $\hat{\theta}$ obtained here $\left(0.3317^{\circ} \mathrm{C}\right)$ is different to that obtained for the triangular signal $\left(0.4043^{\circ} \mathrm{C}\right)$. As a general result, the mean value between the maximum and minimum temperature variations is different from zero in the steady-state regime and depends on the type of loading signal.

Test \#3 corresponds to the same stretch ratio amplitude as for the previous tests, but the strain ratio $R_{\varepsilon}$ is now equal to $1 / 2$ instead of 0 . For isentropic materials that exhibit linear strain-stress elastic relationship, the result would be the same for any tests with same stretch amplitude: the stabilized value of $\hat{\theta}$ would be null. In the case of hyperelastic materials, the conclusion is different: the (non-null) stabilized value of $\hat{\theta}$ depends on the considered zone of strain-stress relationship. For the present simulations, $\hat{\theta}$ equates $0.2819^{\circ} \mathrm{C}$ for $\lambda$ oscillating between 1 and 3 , and $0.3317^{\circ} \mathrm{C}$ for $\lambda$ oscillating between 2 and 4 .
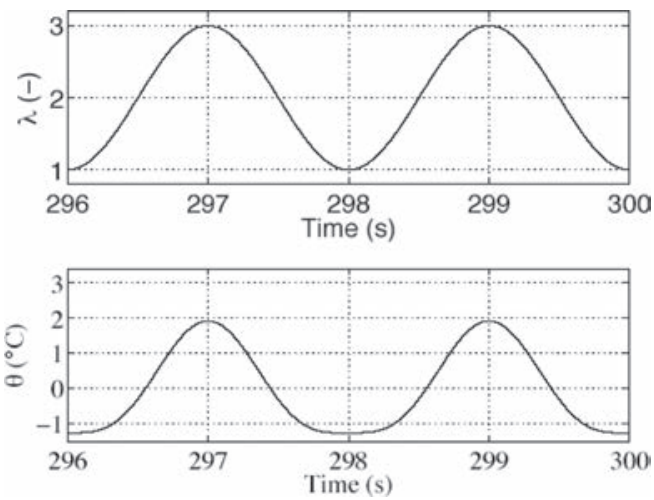

Figure 2. Last two cycles of Test \#2.
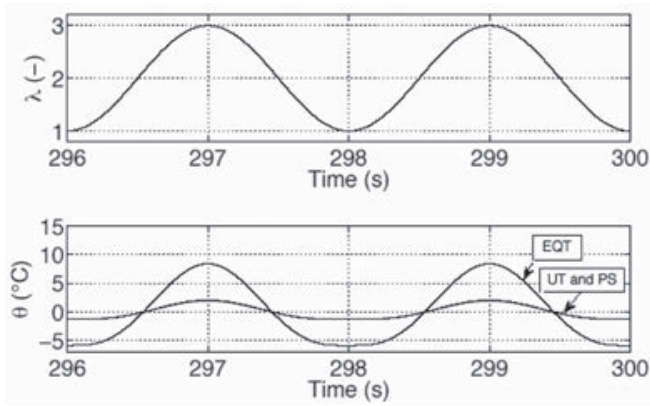

Figure 3. Last two cycles of Tests \#1, \#4 and \#5.

\subsection{Effect of the multiaxiality of the mechanical state}

Tests \#1, \#4 and \#5 enable us to investigate the effect of three loading conditions, Uniaxial Tension (UT), Equibiaxial Tension (EQT) and Pure Shear (PS), respectively, classically used to characterize the mechanical response of rubber-like materials. Figure 3 groups the three stabilized thermal responses. Eq. 6 with $B=-0.5$ and $B=1$ shows that UT and PS exhibit very close values of heat sources, while the heat sources for EQT are approximately twice greater. As a result, the temperature variations for the EQT case strongly differ from the UT and PS cases. For the present numerical simulations, the following results are found in the steady-state regime: $\hat{\theta}=0.3317^{\circ} \mathrm{C}$ for $\mathrm{UT}, 0.3287^{\circ} \mathrm{C}$ for PS and $1.2508^{\circ} \mathrm{C}$ for EQT.

\section{CONCLUSION}

This paper has investigated the thermal stabilization of rubber-like material under cyclic loading 
conditions at ambient temperature. Results have shown that the mean value between the maximum and the minimum temperature variations differs from zero in the steady-state regime. This is due to the particular evolution of the heat source in time, more especially to the fact that the heat source depends on both the strain and the strain rate. This phenomenon is observed while the temporal integration of the heat source is null over each cycle and no mechanical dissipation occurs. This effect clearly distinguishes rubber from most of the other materials, whose thermoelastic response is driven by an isentropic coupling.

The special thermosensitivity of rubber engenders many consequences, in particular for fatigue prediction. Indeed, the stabilized mean value between the maximum and minimum temperature variations during fatigue tests does not reflect only fatigue damage or self-heating, since the entropic coupling leads also to a value that is not equal to zero.

\section{REFERENCES}

Balandraud, X., Ernst, E. \& Soos, E. 1999. Rheological phenomena in shape memory alloys. C. R. Acad. Sci., Ser. IIb Mec 327:33-39.

Balandraud, X., Chrysochoos, A., Leclercq, S. \& Peyroux, R. 2001. Influence of the thermomechanical coupling on the propagation of a phase change front. C R Acad Sci-Series IIB-Mechanics 329:621-626.

Barick, A.K. \& Tripathy, D.K. 2010. Thermal and dynamic mechanical characterization of thermoplastic polyurethane/organoclay nanocomposites prepared by melt compounding. Materials Science and Engineering A 527:812-823.

Barmouz, M., Seyfi, J., Kazem Besharati Givi, M., Hejazi, I. \& Davachi, S.M. 2011. A novel approach for producing polymer nanocomposites by in-situ dispersion of clay particles via friction stir processing. Materials Science and Engineering A 528:3003-3006.

Berthel, B., Chrysochoos, A., Wattrisse, B. \& Galtier, A. 2008. Infrared image processing for the calorimetric analysis of fatigue phenomena. Experimental Mechanics 48:79-90.

Boulanger, T., Chrysochoos, A., Mabru, C. \& Galtier, A. 2004. Calorimetric analysis of dissipative and thermoelastic effects associated with the fatigue behavior of steels. International Journal of Fatigue 26:221-229.

Chadwick, P. \& Creasy, C.M.F. 1984. Modified entropic elasticity of rubberlike materials. Journal of Mechanics and Physics of Solids 32:337-357.

Chrysochoos, A., Pham, H. \& Maisonneuve, O. 1996. Energy balance of thermoelastic martensite transformation under stress. Nuclear Engineering Design 162:1-12.

Chrysochoos, A. \& Louche, H. 2000. An infrared image processing to analyse the calorific effects accompanying strain localisation. Int J Eng Sci 38:1759-1788.

Chrysochoos, A. \& Louche, H. 2001. Thermal and dissipative effects accompanying luders band propagation. Mat Sci Eng A 307:15-22.
Chrysochoos, A., Wattrisse, B., Muracciole, J.M. \& El Kaim, Y. 2009. Fields of stored energy associated with localized necking of steel. J Mech of Mater Struct 4:245-262.

Diani, J., Fayolle, B. \& Gilormini, P. 2009. A review on the mullins effect. European Polymer Journal 45:601-612.

Dumoulin, S., Louche, H., Hopperstad, O.S. \& Borvik, T. 2010. Heat sources, energy storage and dissipation in high-strength steels: Experiments and modelling. European Journal of Mechanics A-Solids 29:461-474.

Fletcher, W.P. \& Gent, A.N. 1953. Non-linearity in the dynamic properties of vulcanised rubber compounds. Trans Inst Rubber Ind 29:266-280.

Gough, J. 1805. Proc Lit and Phil Soc Manchester, 2nd, ser. 1, page 288 .

Huneau, B. 2011. Strain-induced crystallization of natural rubber: a review of x-ray diffraction investigations. Rubber Chemistry and Technology 84:425-452.

Joule, J.P. 1884. India-rubber, IS33 in: The Scientific Papers of James Prescott Joule, volume 1. The Physical Society of London, Taylor and Francis, red lion court, fleet street.

Le Cam, J.B. 2005. Endommagement en fatigue des élastomères. $\mathrm{PhD}$ thesis, Université de Nantes, Ecole Centrale de Nantes.

Le Cam, J.B. \& Toussaint, E. 2008. Volume variation in stretched natural rubber: competition between cavitation and stress-induced crystallization. Macromolecules 41:7579-7583.

Le Cam, J.B. \& Toussaint, E. 2009. Cyclic volume changes in rubbers. Mechanics of Materials 41:898-901.

Lemaitre, J. \& Chaboche, J.L. 1990. Mechanics of Solids Materials. Cambridge University Press, Cambridge.

Maquin, F. \& Pierron, F. 2009. Heat dissipation measurements in low stress cyclic loading of metallic materials: From internal friction to micro-plasticity. Mechanics of Materials 41:928-942.

Marckmann, G., Verron, E., Gornet, L., Chagnon, G., Charrier, P. \& Fort P. 2002. A theory of network alteration for the Mullins effect. Journal of the Mechanics and Physics of Solids 50:2011-2028.

Mars, W.V. \& Fatemi, A. 2002a. A literature survey on fatigue analysis approaches for rubber. International Journal of Fatigue 24:949-961.

Mars, W.V. 2002b. Cracking energy density as a predictor of fatigue life under multiaxial conditions. Rubber Chemistry and Technology 75:1-17.

Maugin, G.A. 1999. The Thermodynamics of Nonlinear Irreversible Behaviors: an Introduction, volume 27 of Series A. World scientific series on Nonlinear Science.

Meunier, L., Chagnon, G., Favier, D., Orgéas, L. \& Vacher, P. 2008. Experimental and numerical study of the mechanical behaviour of an unfilled silicone rubber. Polymer Testing 27:765-777.

Mullins, L. 1948. Effect of stretching on the properties of rubber. Rubber Chemistry and Technology 21:281-300.

Payne, A.R. 1962. The dynamic properties of carbon black-loaded natural rubber vulcanizates. part I. J Appl Phys 6(19):57-63.

Saintier, N., Cailletaud, G. \& Piques, R. 2006. Multiaxial fatigue life prediction for a natural rubber. International Journal of Fatigue 28:530-539. 
Stringfellow, R. \& Abeyaratne, R. 1989. Cavitation in an elastomer: Comparison of theory with experiment. Materials Science and Engineering A 112:127-131.

Toki, S., Fujimaki, T. \& Okuyama, M. 2000. Strain-induced crystallization of natural rubber as detected real-time by wide-angle $\mathrm{x}$-ray diffraction technique. Polymer 41:5423-5429.

Toki, S., Sics, I., Ran, S., Liu, L., Hsiao, B.S., Murakami, S., Senoo, K. \& Kohjiya, S. 2002. Macromolecules 35:6578-6584.

Toussaint, E., Balandraud, X., Le Cam, J.B. \& Grédiac M. 2012. Combining displacement, strain, temperature and heat source fields to investigate the thermomechanical response of an elastomeric specimen subjected to large deformations. Polymer Testing 31:916-925.
Trabelsi, S., Albouy, P.A. \& Rault, J. 2002. Stress-induced crystallization around a crack tip in natural rubber. Macromolecules 35:10054-10061.

Trabelsi, S., Albouy, P.A. \& Rault, J. 2003. Effective local deformation in stretched filled rubber. Macromolecules 36:9093-9099.

Treloar, L.R.G. 1943. The elasticity of a network of long chain molecules (I and II). Transactions of the Faraday Society 39:36-64; 241-246. 